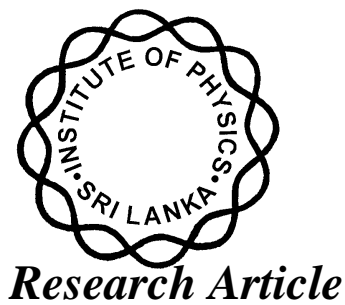

INSTITUTE OF PHYSICS - SRI LANKA

\title{
Solutions of Klein-Gordon equation for the modified central complex potential in the symmetries of noncommutative quantum mechanics
}

\author{
Abdelmadjid Maireche* \\ Laboratory of Physics and Material Chemistry, Physics department, Sciences Faculty, \\ University of M'sila- Algeria.
}

\begin{abstract}
We explore the new analytical solutions for both bound and the new masses of mesons of the Klein-Gordon equation with the modified central complex potential, which describes the heavy-light $Q \bar{q}, \quad(Q=c, q=u / d, s)$ mesons and the quarkonium system $q \bar{q},(q=c, b, s)$ via the standard Bopp's shift method and standard perturbation theory.We have obtained the energy eigenvalues of the ground state $E_{n c}^{(0)}(a, b, 0, j, l, m)$, the first excited state $E_{n c}^{(1)}(a, b, 1, j, l, m)$ and $p^{\text {th }}$ the excited state $E_{n c}^{(p)}(a, b, p, j, l, m)$ in terms of the shift energy $\left(\Delta E_{c c}(0, j, l, s, m), \Delta E_{c c}(1, j, l, s, m)\right.$ and $\left.\Delta E_{c c}(p, j, l, s, m)\right)$ and $\left(E_{0 l}, E_{1 l}\right.$ and $\left.E_{n l}\right)$ of ordinary relativistic quantum mechanics. In addition to the parabolic cylinder functions, the Gamma function, the discreet atomic quantum numbers $(j, l, s, m)$, the potential parameters $(a$ and $b)$ and the noncommutativity parameters $(\theta$ and $\sigma)$. In the second part of the research, we will apply the obtained results to calculate the new masses of the mentioned previously mesons in the symmetries of the relativistic three-dimensional noncommutative quantum mechanics. Moreover, some important special cases in the context of the symmetries of the relativistic three-dimensional noncommutative quantum mechanics are treated.
\end{abstract}

Keywords: Klein-Gordon equation; central complex potential; noncommutative space phase; Bopp's shift method.

* Corresponding author:

abmaireche@gmail.com and abdelmadjid.maireche@univ-msila.dz

https://orcid.org/0000-0002-8743-9926 


\section{INTRODUCTION}

An adequate understanding of the quark behavior of two interacting quarks as a function of their relative positions is very pertinent in different fields of sub-atomic and elementary particles using various potential schemes. Recently, there has been a great interest in obtaining quark potential energy functions governing the interaction of twoquarks (mesons model) ${ }^{1-10}$. In particular, the complex potentials such as exponential type complex and non-Hermitian potentials, generalized Hulthén potential in complex quantum mechanicsand central complex potentials $V(r)=i a r+b / r$ were played a crucial role in particle physics as well as in nuclear physics ${ }^{11-13}$. In addition, this potential is suggested as a quarkonium physics or quark-antiquark interaction potential for studying the masses of the heavy-light $Q \bar{q},(Q=c, q=u / d, s)$ mesons and the quarkonium system $q \bar{q},(q=c, b, s)$ in relativistic three-dimensional noncommutative quantum mechanics, in which the potential satisfied the features of quantum chromodynamics theory of strong interaction ${ }^{14}$. In this paper, the recent progress made by authors V. K. Srivastava and S. K. Rose to motivate us in their studies ${ }^{13}$.

In this paper, motivated bymany various recent studies for example the nonrenormalizable of the electroweak interaction,quantum gravity, string theory, the noncommutative relativistic quantum mechanics has attracted much attentionto physical researchers ${ }^{15-21}$.

This paper aims to understand the central complex potential in large space known by relativistic noncommutative quantum mechanics to achieve a more accurate physical vision so that this study becomes valid in the field of nanotechnology. On the other hand, to explore the possibility of creating new applications and more profound interpretations in the sub-atomics and nano scales using a new version of the modified effective relativistic central complex potential, which has the following form:

$$
\begin{aligned}
& V_{e f f}^{c c}(r)=2(E+M)(i a r+b / r)+\frac{l(l+1)}{2 r^{2}} \rightarrow \\
& V_{e f f-n c}^{c c}(\hat{r})=V_{e f f}^{c c}(r)+\left[\frac{l(l+1)}{r^{4}}-(E+M)\left(\frac{i a}{r}+\frac{b}{r^{3}}\right)\right] \overrightarrow{\mathbf{L}} \vec{\Theta}
\end{aligned}
$$

here $i^{2}=-1, a$ and $b$ are the real positive potential parameters and $\mathrm{r}$ is the interquark distance while the coupling $\overrightarrow{\mathbf{L}} \vec{\Theta}$ is interpreted as the interaction between the angular momentum operator and noncommutativity properties of space-space. In this paper, we have introduced the modified central complex potential, which takes the following form:

$$
V_{c c}(\hat{r})=i a r+b / r-\left(\frac{i a}{2 r}-\frac{b}{2 r^{3}}\right) \overrightarrow{\mathbf{L}} \vec{\Theta}+O\left(\Theta^{2}\right)
$$

It should be noted that the noncommutativity was introduced firstly by W.Heisenberg 22 in 1930 and then by H.Syndre 23 in 1947. Up to our knowledge, no attempts to study the modified central complex potential using Bopp's shift method. The new structure of the relativistic noncommutative relativistic quantum mechanicsbased on new $\mathrm{NC}$ canonical 
commutations relations in Schrödinger, Heisenberg and interactions pictures, respectively, as follows ${ }^{24-29}$ (Throughout this paper, the natural units $c=\hbar=1$ will be used):

$$
\left\{\begin{array}{l}
\left\lfloor\hat{x}_{i}^{*}, \hat{p}_{j}\right\rfloor=\left\lfloor\hat{x}_{i}(t)^{*}, \hat{p}_{j}(t)\right\rfloor=\left\lfloor\hat{x}_{I i}(t)^{*}, \hat{p}_{I j}(t)\right\rfloor=i \delta_{i j} \\
{\left[\begin{array}{c}
\hat{x}_{i}, \hat{x}_{j} \\
{[}
\end{array}=\left[\hat{x}_{i}(t)^{*}, \hat{x}_{j}(t)\right]=\left[\hat{x}_{I i}(t)^{*}, \hat{x}_{I j}(t)\right]=i \theta_{i j}\right.}
\end{array}\right.
$$

where the indices $(i, j \equiv 1,2,3)$ while $\left|A^{*}, B\right| \equiv A^{*} B-B^{*} A$, for any two operators $A$ and $B$. However, the new operators $\hat{\xi}_{i}^{H}-(t)=\left(\hat{x}_{i} \vee \hat{p}_{i}\right)(t)$ and $\hat{\xi}_{i}^{I}(t)=\left(\hat{x}_{I i} \vee \hat{p}_{I i}\right)(t)$ in (Heisenberg and interaction pictures, respectively) are depending on the corresponding new operator $\hat{\xi}_{i}^{S}=\hat{x}_{i} \vee \hat{p}_{i}$ in Schrödinger picture from the following projections relations:

$\left\{\begin{array}{l}\xi_{i}^{H}(t)=\exp \left(i \hat{H}_{c c} T\right) \xi_{i}^{S} \exp \left(-i \hat{H}_{c c} T\right) \\ \xi_{i}^{I}(t)=\exp \left(i \hat{H}_{o}^{c c} T\right) \xi_{i}^{S} \exp \left(-i \hat{H}_{o}^{c c} T\right)\end{array} \Rightarrow\left\{\begin{array}{l}\hat{\xi}_{i}^{H}(t)=\exp \left(i \hat{H}^{c c} T\right) * \hat{\xi}_{i}^{S} * \exp \left(-i \hat{H}^{c c} T\right) \\ \hat{\xi}_{i}^{I}(t)=\exp \left(i \hat{H}_{n c o}^{c c} T\right) * \hat{\xi}_{i}^{S} * \exp \left(-i \hat{H}_{n c o}^{c c} T\right)\end{array}\right.\right.$

here $T \equiv t-t_{0}$ while $\xi_{i}^{S}=\left(x_{i} \vee p_{i}\right), \xi_{i}^{H}(t)=\left(x_{i} \vee p_{i}\right)(t)$ and $\hat{\xi}_{i}^{I}(t)=\left(x_{I i} \vee p_{I i}\right)(t)$ are the three representations in relativistic quantum mechanics. The dynamics of new systems $\left(\frac{\mathrm{d} \hat{\xi}_{i}^{H}(t)}{\mathrm{dt}}\right.$ and $\left.\frac{\mathrm{d} \xi_{i}^{I}(t)}{\mathrm{dt}}\right)$ are described from the following motion equations in relativistic noncommutative relativistic quantum mechanics:

$$
\left\{\begin{array}{l}
\frac{\mathrm{d} \xi_{i}^{H}(t)}{\mathrm{dt}}=\left[\xi_{i H}(t), \hat{H}_{c c}\right]+\frac{\partial \xi_{i}^{H}(t)}{\partial \mathrm{t}} \Rightarrow \frac{\mathrm{d} \hat{\xi}_{i}^{H}(t)}{\mathrm{dt}}=\left[\hat{\xi}_{i}^{H}(t)^{*}, \hat{H}_{n c o}^{c c}\right]+\frac{\partial \hat{\xi}_{i}^{H}(t)(t)}{\partial \mathrm{t}} \\
\frac{\mathrm{d} \xi_{i}^{I}(t)}{\mathrm{dt}}=\left[\xi_{i}^{I}(t), \hat{H}_{c c}\right]+\frac{\partial \xi_{i}^{I}(t)}{\partial \mathrm{t}} \Rightarrow \frac{\mathrm{d} \hat{\xi}_{i}^{I}(t)}{\mathrm{dt}}=\left[\hat{\xi}_{i}^{I}(t)^{*}, \hat{H}_{n c o}^{c c}\right]+\frac{\partial \hat{\xi}_{i}^{I}(t)(t)}{\partial \mathrm{t}}
\end{array}\right.
$$

where $\hat{H}_{c c}\left(\hat{H}_{o}^{c c}\right)$ and $\hat{H}_{n c o}^{c c}\left(\hat{H}_{n c o}^{c c}\right)$ represent the global quantum Hamiltonian (the unperturbed Hamiltonian)operators for complex potential and modified complex potential in the relativistic quantum mechanics and its extension, respectively. The very small parameter $\theta^{i j}$ (compared to the energy) are elements of theantisymmetric real matrix and $(*)$ denote the Weyl Moyal star product, which is generalized between two arbitrary functions $(f g)(x)$ to the new form $\hat{f}(\hat{x}) \hat{g}(\hat{x}) \equiv(f * g)(x)$ in relativistic threedimensional noncommutative quantum mechanicssymmetries ${ }^{30-42}$ :

$$
(f g)(x) \rightarrow(f * g)(x)=\exp \left(i \theta_{i j} \partial_{x_{i}} \partial_{x_{j}}\right) f\left(x_{i}\right) g\left(x_{j}\right) \cong f g(x)-\left.\frac{i}{2} \theta^{i j} \partial_{i}^{x} f \partial_{j}^{x} g\right|_{x_{i}=x_{j}}+O\left(\theta^{2}\right)
$$

which $O\left(\theta^{2}\right)$ stands for the second and higher-order terms of the infinitesimal parameter $\theta$. The second in the above equation presents the effects of (space-space) 
noncommutativity properties. The objective of this study is two-fold; firstly, we solve the modified Klein-Gordon equation with the modified central complex potential using the Bopp's shift method and standard perturbation theory, we apply the energy equation obtained to study atomic behavior in some selected the heavy-light $Q \bar{q}$, $(Q=c, q=u / d, s)$ mesons, and the quarkonium system $q \bar{q},(q=c, b, s)$ of this potential. While we deal in the third part of some important special cases. The scheme of our research article is as follows. Section 1 has the introduction, a brief description of the eigenfunctions and the energy eigenvalues for the central complex potential in the relativistic quantum mechanics is reviewed in section 2 . In section 3 , the modified radial Klein-Gordon equation with the modified central complex potential is solved via the standard Bopp's shift method and the standard perturbation theory. In the next section, we apply our results to calculating the new masses of heavy-light $Q \bar{q}$, $(Q=c, q=u / d, s)$ mesons and the quarkonium system $q \bar{q},(q=c, b, s)$, then, we present a special case of the potential under consideration.Finally, we discuss some particulars casesin section 5 before the conclusion in section 5 .

\section{OVERVIEW OF THE RELATIVISTIC ENERGY LEVELS AND WAVE FUNCTION FOR CENTRAL COMPLEX POTENTIAL}

As already mentioned, we aim to obtain the relativistic spectrum of the modified central complex potential $V_{c c}(r)=i a r+b / r$ in a three-dimensional relativistic noncommutative quantum equation. In spherical coordinates, the Klein-Gordon equation with the scalar potential $S_{c c}(r)$ and the vector potential $V_{c c}(r)$ is given by $(c=\hbar=1)$ :

$$
\left(\nabla^{2}-\left(M-S_{c c}(r)\right)^{2}+\left(E-V_{c c}(r)\right)^{2}\right) \Psi(r, \theta, \varphi)=0
$$

If the wave function is selected as $\Psi(r, \theta, \varphi)=R_{p l}(r) Y_{l}^{m}(\theta, \varphi)$ and after the necessary calculations are done, the radial part of the Klein-Gordon equation $R_{l}(r)$ is obtained as the following form:

$$
\left\{\frac{d^{2}}{d r^{2}}+\frac{2}{r} \frac{d}{d r}-\left(M^{2}-E_{p l}^{2}\right)-2\left(E V_{c c}(r)+M S_{c c}(r)\right)+V_{c c}^{2}(r)-S_{c c}^{2}(r)-\frac{l(l+1)}{r^{2}}\right\} R_{p l}(r)=0
$$

where $l$ is eigenvalues of the angular momentum. For removing the derivation of the first order, we introduce $R_{p l}(r)=\frac{U_{p l}(r)}{r}$, thus Eq. (7) become in the case of equal scalar and vector potentials $V_{c c}(r)=S_{c c}(r)$ :

$$
\left\{\frac{d^{2}}{d r^{2}}+\left(E_{p l}^{2}-M^{2}\right)-2(E+M)(i a r+b / r)-\frac{l(l+1)}{r^{2}}\right\} U_{p l}(r)=0
$$

Based on the ref. [13], the complete wave function is the following: 


$$
\Psi^{(p)}(r, \theta, \varphi)=\left\{\begin{array}{cc}
a_{0} r^{\nu-1} \exp \left(-\frac{1}{2} \alpha r^{2}-\beta_{0} r\right) Y_{l}^{m}(\theta, \varphi) & \text { for } \quad p=0 \\
\left(a_{0} r^{\nu-1}+a_{1} r^{v}\right) \exp \left(-\frac{1}{2} \alpha r^{2}-\beta_{1} r\right) Y_{l}^{m}(\theta, \varphi) & \text { for } \quad p=1 \\
\sum_{n=0}^{\infty} a_{n} r^{n+v} \exp \left(-\frac{1}{2} \alpha r^{2}-\beta_{p} r\right) Y_{l}^{m}(\theta, \varphi) & \text { for } \quad p
\end{array}\right.
$$

Where $a_{i}$ can be determined by the normalization condition, $v=v_{ \pm}=-1 / 2 \pm \sqrt{(l+1 / 2)^{2}-b^{2}}, \alpha=\sqrt{a}$ and $\beta_{p}=\sqrt{a} E_{p}$.Also, the energy $E_{p l}$ of the relativistic potential obtained from the following relation:

$$
\begin{aligned}
& E_{0 l}=\left(M^{2}-2 a b+\left(2 v_{ \pm}+1\right) /(a+1)\right)^{1 / 2} \\
& E_{1 l}=\left(M^{2}-2 a b+\left(2 v_{ \pm}+3\right) /(a+1)\right)^{1 / 2} \\
& E_{p l}= \pm\left(M^{2}-2 a b+\left(2 p+2 v_{ \pm}+1\right) /(a+1)\right)^{1 / 2}
\end{aligned}
$$

It is a worth motion that the potential used in the paper should satisfy the Coulomb force proportional to $\left(-b / r^{2}\right)$ which expresses the asymptotic nature of the strong interaction at short distances and the confinement forceproportional to $(-i a)$ produced from the linear part for describing the interaction between light and heavy mesons at large distances. This physical behavior is quite similarto the Cornell potential ${ }^{43-44}$.It can also be said that the physical behavior of the studied potential, in ordinary nonrelativistic quantum mechanics, is similar to the trigonometric Rosen-Morse potential (suggested as a quark-antiquark interaction potential)which is as a function of distance $r$ for the exact and approximate potential on the second page of the Ref. ${ }^{6}$.

\section{SOLUTION OF MODIFIED KLEIN-GORDON EQUATION FOR SOLUTION OF MKG MODIFIED CENTRAL COMPLEX POTENTIAL}

In this section, we shall give an overview or a brief preliminary for the modified central complex potential in relativistic three-dimensional noncommutative quantum mechanicssymmetries. To perform this task the physical form of modified Klein-Gordon equation it is necessary to apply the notion of the Weyl Moyal star product on the differential equation satisfied by the radial wave function $U_{p l}(r)$ in Eq. (7), thus, the radial wave function $U_{p l}(r)$ in relativistic three-dimensional noncommutative quantum mechanicssymmetries become $25-29$ : 


$$
\begin{aligned}
& \left\{\frac{d^{2}}{d r^{2}}+\left(E^{2}-M^{2}\right)-2(E+M) V_{c c}(r)-\frac{l(l+1)}{r^{2}}\right\} U_{p l}(r)=0 \Rightarrow \\
& \left\{\frac{d^{2}}{d r^{2}}+\left(E^{2}-M^{2}\right)-2(E+M) V_{c c}(r)-\frac{l(l+1)}{r^{2}}\right\} * U_{p l}(r)=0
\end{aligned}
$$

The Bopp's shift method has been successfully applied to the relativistic noncommutative quantum mechanics and nonrelativistic noncommutative quantum mechanics problems using a modified Dirac equation, modified Klein-Gordon equation and modified Schrödinger equation. This method has produced very promising results for several situations having physical, chemical interest ${ }^{30-36}$. The method reduces three modified fundamental equations (modified Dirac equation, modified Klein-Gordon equation and modified Schrödinger equation) to the (Dirac equation, Klein-Gordon equation and Schrödinger equation), respectively, under the simultaneous translation in space. It based on the following new commutator ${ }^{34-42}$ :

$$
\left[\hat{x}_{\mu}, \hat{x}_{v}\right]=\left[\hat{x}_{\mu}(t), \hat{x}_{v}(t)\right]=i \theta_{\mu v}
$$

The new generalized positions and momentum coordinates $\left(\hat{x}_{\mu}, \hat{p}_{v}\right)$ in the relativistic three-dimensional noncommutative quantum mechanicsare defined in terms of the commutative counterparts $\left(x_{\mu}, p_{v}\right)$ in quantum mechanics via, respectively ${ }^{41-42,45-52}$ :

$$
\left(x_{\mu}, p_{v}\right) \Rightarrow\left(\hat{x}_{\mu}, \hat{p}_{v}\right)=\left(x_{\mu}-\frac{\theta_{\mu v}}{2} p_{v}, p_{\mu}\right)
$$

The above equation allows us to obtain the operator $r^{2} \Rightarrow \hat{r}^{2}=r^{2}-\overrightarrow{\mathbf{L}} \vec{\Theta}$ in the relativistic three-dimensional noncommutative quantum mechanicssymmetries. The two couplings $\overrightarrow{\mathbf{L}} \vec{\Theta}$ equal $\left(L_{x} \Theta_{12}+L_{y} \Theta_{23}+L_{z} \Theta_{13}\right)$ and $\left(L_{x}, L_{y}\right.$ and $\left.L_{z}\right)$ are the three components of the angular momentum operator $\overrightarrow{\mathbf{L}}$ while $\Theta_{\mu \nu}=\theta_{\mu \nu} / 2$ the new parameter $\Theta_{\mu v}$ equals ${ }^{40-42,45-46} \theta_{\mu \nu} / 2$. Thus, the reduced like Schrödinger equation (without star product) can be written as:

$$
\begin{aligned}
& \left\{\frac{d^{2}}{d r^{2}}+\left(E^{2}-M^{2}\right)-2(E+M) V(r)-\frac{l(l+1)}{r^{2}}\right\} * U_{p l}(r)=0 \Rightarrow \\
& \left\{\frac{d^{2}}{d r^{2}}+\left(E^{2}-M^{2}\right)-2(E+M) V(\hat{r})-\frac{l(l+1)}{\hat{r}^{2}}\right\} U_{p l}(r)=0
\end{aligned}
$$


The new operator $V_{c c}(\hat{r})$ can be expressed as ${ }^{25-29}$ :

$$
V_{c c}(\hat{r}) \equiv V_{c s}\left(\sqrt{\left(x_{\mu}-\frac{\theta_{\mu \nu}}{2} p_{v}\right)\left(x^{\mu}-\frac{\theta^{\mu \alpha}}{2} p_{\alpha}\right)}\right)=V_{c c}(r)-\frac{\overrightarrow{\mathbf{L}} \vec{\Theta}}{2 r} \frac{\partial V_{c s}(r)}{\partial r}+O\left(\Theta^{2}\right)
$$

We have $\frac{\partial V_{c c}(r)}{\partial r}=i a-\frac{b}{r^{2}}$ and $\frac{1}{\hat{r}^{2}} \approx \frac{1}{\hat{r}^{2}}+\frac{\overrightarrow{\mathbf{L}} \vec{\Theta}}{r^{4}}+O\left(\Theta^{2}\right)$, allows us to write the modified radial part of the modified Klein-Gordon equation in the relativistic three-dimensional noncommutative quantum mechanics symmetries:

$$
\left\{\frac{d^{2}}{d r^{2}}+\left(E^{2}-M^{2}\right)-2(E+M) V(r)-\frac{l(l+1)}{r^{2}}-\left[\frac{l(l+1)}{r^{4}}-(E+M)\left(\frac{i a}{r}+\frac{b}{r^{3}}\right)\right] \overrightarrow{\mathbf{L}} \vec{\Theta}\right\} U_{p l}(r)=0(15)
$$

Moreover, to illustrate this equation in a simple mathematical way, it is useful to enter the following symbols $V_{\text {eff }}^{c c}(r)(r)=2(E+M)(i a r+b / r)+\frac{l(l+1)}{r^{2}}$ and $E_{e f f}^{c c}=M^{2}-E^{2}$, thus the radial equation (15) becomes:

$$
\left(\frac{d^{2}}{d r^{2}}-\left(E_{e f f}^{c c}+V_{e f f}^{c c}(r)(r)+V_{p e r t}^{c c}(r)\right)\right) U_{p l}(r)=0
$$

with:

$$
V_{p e r t}^{c c}(r)=\left(\frac{l(l+1)}{r^{4}}-(E+M)\left(\frac{i a}{r}+\frac{b}{r^{3}}\right)\right) \overrightarrow{\mathbf{L}} \vec{\Theta}
$$

The additive part of the effective potential is proportional to the infinitesimal vector $\vec{\Theta}=\Theta_{11} e_{x}+\Theta_{12} e_{y}+\Theta_{13} e_{z}$.Thus, we can consider $V_{p e r t}^{c c}(r)(r)$ as perturbation terms compared with the parent potential (effective potential operator) $V_{e f f}^{c c}(r)(r)$ in the relativistic three-dimensional noncommutative quantum mechanics symmetries. The purpose here is to give a complete prescription for determining the energy level of the ground state, the first excited state and $p^{\text {th }}$ excited state, by applying the perturbative theory, in the case ofthe relativistic noncommutative relativistic quantum mechanics.In the first-order perturbation theory the expectation value of $r^{-1}, r^{-3}$ and $r^{-4}$ concerning the exact solution of Eq. (7), is given by: 


$$
\begin{aligned}
\left\langle 0, l, m\left|r^{-1}\right| 0, l, m\right\rangle & =a_{0} \int_{0}^{+\infty} r^{2 v-1} \exp \left(-\alpha r^{2}-2 \beta_{0} r\right) d r \\
\left\langle 0, l, m\left|r^{-3}\right| 0, l, m\right\rangle & =a_{0} \int_{0}^{+\infty} r^{2 v-3} \exp \left(-\alpha r^{2}-2 \beta_{0} r\right) d r \\
\left\langle 0, l, m\left|r^{-4}\right| 0, l, m\right\rangle & =a_{0} \int_{0}^{+\infty} r^{2 v-4} \exp \left(-\alpha r^{2}-2 \beta_{0} r\right) d r \\
\left\langle 1, l, m\left|r^{-1}\right| 1, l, m\right\rangle & =\int_{0}^{+\infty}\left(a_{0} r^{\nu-1}+a_{1} r^{v}\right)^{2} \exp \left(-\alpha r^{2}-2 \beta_{1} r\right) r d r \\
\left\langle 1, l, m\left|r^{-3}\right| 1, l, m\right\rangle & =\int_{0}^{+\infty}\left(a_{0} r^{\nu-1}+a_{1} r^{v}\right)^{2} \exp \left(-\alpha r^{2}-2 \beta_{1} r\right) r^{-1} d r \\
\left\langle 1, l, m\left|r^{-4}\right| 1, l, m\right\rangle & =\int_{0}^{+\infty}\left(a_{0} r^{\nu-1}+a_{1} r^{\nu}\right)^{2} \exp \left(-\alpha r^{2}-2 \beta_{1} r\right) r^{-2} d r
\end{aligned}
$$

and

$$
\begin{aligned}
& \left\langle p, l, m\left|r^{-1}\right| p, l, m\right\rangle=\int_{0}^{+\infty}\left(\sum_{n=0}^{\infty} a_{n} r^{n+v}\right)^{2} \exp \left(-\alpha r^{2}-2 \beta_{p} r\right) r d r \\
& \left\langle p, l, m\left|r^{-3}\right| p, l, m\right\rangle=\int_{0}^{+\infty}\left(\sum_{n=0}^{\infty} a_{n} r^{n+v}\right)^{2} \exp \left(-\alpha r^{2}-2 \beta_{p} r\right) r^{-1} d r \\
& \left\langle p, l, m\left|r^{-4}\right| p, l, m\right\rangle=\int_{0}^{+\infty}\left(\sum_{n=0}^{\infty} a_{n} r^{n+v}\right)^{2} \exp \left(-\alpha r^{2}-2 \beta_{p} r\right) r^{-2} d r
\end{aligned}
$$

Allows us to simplify Eqs. (18) and (19) to the new form:

$$
\begin{aligned}
& \left\langle 0, l, m\left|r^{-1}\right| 0, l, m\right\rangle=a_{0} \int_{0}^{+\infty} r^{2 v-1} \exp \left(-\alpha r^{2}-2 \beta_{0} r\right) d r \\
& \left\langle 0, l, m\left|r^{-3}\right| 0, l, m\right\rangle=a_{0} \int_{0}^{+\infty} r^{2 v-2-1} \exp \left(-\alpha r^{2}-2 \beta_{0} r\right) d r \\
& \left\langle 0, l, m\left|r^{-4}\right| 0, l, m\right\rangle=a_{0} \int_{0}^{+\infty} r^{2 v-3-1} \exp \left(-\alpha r^{2}-2 \beta_{0} r\right) d r
\end{aligned}
$$

and 


$$
\begin{aligned}
& \left\langle 1, l, m\left|r^{-1}\right| 1, l, m\right\rangle=\int_{0}^{+\infty}\left(a_{0}{ }^{2} r^{2 v-1-1}+a_{1}{ }^{2} r^{2 v+2-1}+2 a_{0} a_{1} r^{2 v+1-1}\right) \exp \left(-\alpha r^{2}-2 \beta_{1} r\right) r d r \\
& \left\langle 1, l, m\left|r^{-3}\right| 1, l, m\right\rangle=\int_{0}^{+\infty}\left(a_{0}{ }^{2} r^{2 v-2-1}+a_{1}{ }^{2} r^{2 v-1}+2 a_{0} a_{1} r^{2 v-1-1}\right) \exp \left(-\alpha r^{2}-2 \beta_{1} r\right) r^{-1} d r \\
& \left\langle 1, l, m\left|r^{-4}\right| 1, l, m\right\rangle=\int_{0}^{+\infty}\left(a_{0}{ }^{2} r^{2 v-4}+a_{1}{ }^{2} r^{2 v-1-1}+2 a_{0} a_{1} r^{2 v-2-1}\right) \exp \left(-\alpha r^{2}-2 \beta_{1} r\right) r^{-2} d r
\end{aligned}
$$

We have used the orthogonality property of the spherical harmonics $\int Y_{l}^{m}(\theta, \varphi) Y_{l^{\prime}}^{m^{\prime}}(\theta, \varphi) \sin (\theta) d \theta d \varphi=\delta_{l l^{\prime}} \delta_{m m^{\prime}}$. It is convenient to apply the following special integral ${ }^{53}$ :

$$
\int_{0}^{+\infty} x^{\nu-1 .} \exp \left(-\lambda x^{2}-\gamma x\right) d x=(2 \lambda)^{-\frac{v}{2}} \Gamma(v) \exp \left(\frac{\gamma^{2}}{8 \lambda}\right) D_{-v}\left(\frac{\gamma}{\sqrt{2 \lambda}}\right)
$$

Where $D_{-v}\left(\frac{\gamma}{\sqrt{2 \lambda}}\right)$ and $\Gamma(v)$ denote to the parabolic cylinder functions and Gamma function. After straightforward calculations, we can obtain explicit results:

$$
\begin{array}{r}
\left\langle 0, l, m\left|r^{-1}\right| 0, l, m\right\rangle=a_{0}{ }^{2}(2 \alpha)^{-v} \Gamma(2 v) \exp \left(\frac{\beta_{0}{ }^{2}}{2 \alpha}\right) D_{-2 v}\left(\sqrt{\frac{2}{\alpha}} \beta_{0}\right) \\
\left\langle 0, l, m\left|r^{-3}\right| 0, l, m\right\rangle=a_{0}{ }^{2}(2 \alpha)^{-\frac{2 v-2}{2}} \Gamma(2 v-2) \exp \left(\frac{\beta_{0}{ }^{2}}{2 \alpha}\right) D_{-(2 v-2)}\left(\sqrt{\frac{2}{\alpha}} \beta_{0}\right) \\
\left\langle 0, l, m\left|r^{-4}\right| 0, l, m\right\rangle=a_{0}{ }^{2}(2 \alpha)^{-\frac{2 v-3}{2}} \Gamma(2 v-3) \exp \left(\frac{\beta_{0}{ }^{2}}{2 \alpha}\right) D_{-(2 v-3)}\left(\sqrt{\frac{2}{\alpha}} \beta_{0}\right)
\end{array}
$$

and

$$
\begin{aligned}
& \left\langle 1, l, m\left|r^{-1}\right| 1, l, m\right\rangle \exp \left(-\frac{\beta_{1}^{2}}{2 \alpha}\right)=a_{0}^{2}(2 \alpha)^{-\frac{2 v-1}{2}} \Gamma(2 v-1) D_{-(2 v-1)}\left(\varepsilon_{1}\right) \\
& +a_{1}^{2}(2 \alpha)^{-\frac{2 v+2}{2}} \Gamma(2 v+2) D_{-(2 v+2)}\left(\varepsilon_{1}\right)+2 a_{0} a_{1}(2 \alpha)^{-\frac{2 v+1}{2}} \Gamma(2 v+1) D_{-(2 v+1)}\left(\varepsilon_{1}\right) \\
& \left\langle 1, l, m\left|r^{-3}\right| 1, l, m\right\rangle \exp \left(-\frac{\beta_{1}^{2}}{2 \alpha}\right)=a_{0}^{2}(2 \alpha)^{-\frac{2 v-2}{2}} \Gamma(2 v-2) D_{-(2 v-2)}\left(\varepsilon_{1}\right) \\
& +a_{1}^{2}(2 \alpha)^{-\frac{2 v}{2}} \Gamma(2 v) D_{-(2 v)}\left(\varepsilon_{1}\right)+2 a_{0} a_{1}(2 \alpha)^{-\frac{2 v-1}{2}} \Gamma(2 v-1) D_{-(2 v-1)}\left(\varepsilon_{1}\right)
\end{aligned}
$$




$$
\begin{aligned}
& \left\langle 1, l, m\left|r^{-4}\right| 1, l, m\right\rangle \exp \left(-\frac{\beta_{1}^{2}}{2 \alpha}\right)=a_{0}^{2}(2 \alpha)^{-\frac{2 v-4}{2}} \Gamma(2 v-4) D_{-(2 v-4)}\left(\varepsilon_{1}\right) \\
& +a_{1}^{2}(2 \alpha)^{-\frac{2 v-1}{2}} \Gamma(2 v-1) D_{-(2 v-1)}\left(\varepsilon_{1}\right)+2 a_{0} a_{1}(2 \alpha)^{-\frac{2 v-2}{2}} \Gamma(2 v-2) D_{-(2 v-2)}\left(\varepsilon_{1}\right)
\end{aligned}
$$

With $\varepsilon_{1}=\sqrt{\frac{2}{\alpha}} \beta_{1}$. We have two principals cases, the first one corresponds to replace $\overrightarrow{\mathbf{L}} \vec{\Theta}$ by $\Theta \vec{L} \vec{S}\left(\Theta=\sqrt{\Theta_{12}{ }^{2}+\Theta_{23}{ }^{2}+\Theta_{13}{ }^{2}}\right)$, we have chosen the vector $\vec{\Theta}$ parallel to the spin $\vec{S}$ and we replace $\Theta \vec{L} \vec{S}$ by $\frac{\Theta}{2}\left(\vec{J}^{2}-\vec{L}^{2}-\vec{S}^{2}\right)$. The set $\left(H_{s o}^{c c}(r, \Theta), \mathrm{J}^{2}, \mathrm{~L}^{2}, \mathrm{~S}^{2}\right.$ and $\left.J_{z}\right)$ forms a complete of conserved physics quantities, the eigenvalues of the spin-orbit coupling operator are $k(l) \equiv \frac{1}{2}(j(j+1)-l(l+1)-s(s+1)), \quad$ with $|l-s| \leq j \leq|l+s|$. Allows us to obtain the energy shift $\Delta E(0, j, l, s), \Delta E(1, j, l, s)$ and $\Delta E(n, j, l, s)$ due to the spin-orbital complying induced by $V_{p e r t}^{c c}(r)$ in the relativistic three-dimensional noncommutative quantum mechanics symmetries as follows (Starting now we will use the following shorthand notation $\left.\langle A\rangle_{(p, l, m)}=\langle p, l, m|A| p, l, m\rangle\right)$ :

$$
\begin{aligned}
& \Delta E(0, j, l, s)=k(l) \Theta\left(l(l+1)\left\langle r^{-4}\right\rangle_{(0, l, m)}-\left(E_{0}+M\right)\left(i a\left\langle r^{-1}\right\rangle_{(0, l, m)}-b\left\langle r^{-3}\right\rangle_{(0, l, m)}\right)\right) \\
& \Delta E(1, j, l, s)=k(l) \Theta\left(l(l+1)\left\langle r^{-4}\right\rangle_{(1, l, m)}-\left(E_{1}+M\right)\left(i a\left\langle r^{-1}\right\rangle_{(1, l, m)}-b\left\langle r^{-3}\right\rangle_{(1, l, m)}\right)\right) \\
& \Delta E(p, j, l, s)=k(l) \Theta\left(l(l+1)\left\langle r^{-4}\right\rangle_{(p, l, m)}-\left(E_{p}+M\right)\left(i a\left\langle r^{-1}\right\rangle_{(p, l, m)}-b\left\langle r^{-3}\right\rangle_{(p, l, m)}\right)\right)
\end{aligned}
$$

The second case corresponds to replace both $\left(\overrightarrow{\mathbf{L}} \vec{\Theta}\right.$ and $\left.\Theta_{13}\right)$ by $\left(\sigma_{13} B L_{z}\right.$ and $\left.\sigma_{13} B\right)$ in addition to use $\left\langle n, l, m\left|L_{z}\right| n^{\prime}, l^{\prime}, m^{\prime}\right\rangle=m^{\prime} \delta_{n n^{\prime}} \delta_{l l^{\prime}} \delta_{m m^{\prime}}$ (with $-l \leq m \leq+l$ ). Allows us to obtain the energy shift $\Delta E_{c c}(0, m), \Delta E_{c c}(1, m)$ and $\Delta E_{c c}(p, m)$ due to the modified Zeeman effect induced by $V_{\text {pert-cc }}(r)$ in the relativistic three-dimensional noncommutative quantum mechanics symmetries as follows:

$$
\begin{aligned}
& \Delta E_{c c}(0, m)=\sigma\left\{l(l+1)\left\langle r^{-4}\right\rangle_{(0, l, m)}-\left(E_{0}+M\right)\left(i a\left\langle r^{-1}\right\rangle_{(0, l, m)}-b\left\langle r^{-3}\right\rangle_{(0, l, m)}\right)\right) B m \\
& \Delta E_{c c}(1, m)=\sigma\left\{\left((l+1)\left\langle r^{-4}\right\rangle_{(1, l, m)}-\left(E_{1}+M\right)\left(i a\left\langle r^{-1}\right\rangle_{(1, l, m)}-b\left\langle r^{-3}\right\rangle_{(1, l, m)}\right)\right) B m\right. \\
& \Delta E_{c c}(p, m)=\sigma\left\{l(l+1)\left\langle r^{-4}\right\rangle_{(p, l, m)}-\left(E_{p}+M\right)\left(i a\left\langle r^{-1}\right\rangle_{(p, l, m)}-b\left\langle r^{-3}\right\rangle_{(p, l, m)}\right)\right\} B m
\end{aligned}
$$

The superposition principle permitted to deduce the additive energy shift $\Delta E_{c c}(0, j, l, s, m), \Delta E_{c c}(1, j, l, s, m)$ and $\Delta E_{c c}(p, j, l, s, m)$ due to the spin-orbit complying and modified Zeeman effect which induced by $V_{p e r t}^{c c}(r)$ in the relativistic threedimensional noncommutative quantum mechanics symmetries as follows: 


$$
\begin{aligned}
& \Delta E_{c c}(0, j, l, s, m)=(k(l) \Theta+B \sigma m)\left(l(l+1)\left\langle r^{-4}\right\rangle_{(0, l, m)}-\left(E_{0}+M\right)\left(i a\left\langle r^{-1}\right\rangle_{(0, l, m)}-b\left\langle r^{-3}\right\rangle_{(0, l, m)}\right)\right)(28 \\
& \Delta E_{c c}(1, j, l, s, m)=(k(l) \Theta+B \sigma m)\left(l(l+1)\left\langle r^{-4}\right\rangle_{(1, l, m)}-\left(E_{1}+M\right)\left(i a\left\langle r^{-1}\right\rangle_{(1, l, m)}-b\left\langle r^{-3}\right\rangle_{(1, l, m)}\right)\right)(2 \\
& \left.\Delta E_{c c}(p, j, l, s, m)=(k(l) \Theta+B \sigma m)\left(l(l+1)\left\langle r^{-4}\right\rangle_{(p, l, m)}-\left(E_{p}+M\right)\right)\left(i a\left\langle r^{-1}\right\rangle_{(p, l, m)}-b\left\langle r^{-3}\right\rangle_{(p, l, m)}\right)\right)(2
\end{aligned}
$$

When we look to the expressions of effective central complex potential $V_{\text {eff }}^{c c}(r)$ and effective energy $E_{\text {eff }}^{c c}$, it is clear that the energy values $E_{\text {eff }}^{c c}$ have a carry unit of energy, thus we can deduce explicitly the energy of ground state $E^{(0)}(a, b, 0, j, l, m)$, first excited state $E_{n c}^{(1)}(a, b, 1, j, l, m)$ and $p^{\text {th }}$ the excited state $E_{n c}^{(p)}(a, b, n, j, l, m)$ as a function of the shift energy $\left(\Delta E_{c c}(0, j, l, s, m), \Delta E_{c c}(1, j, l, s, m)\right.$ and $\left.\Delta E_{c c}(p, j, l, s, m)\right)$ and $\left(E_{0}, E_{1}\right.$ and $\left.E_{p}\right)$ as follows :

$$
\begin{aligned}
& E_{n c}^{(0)}(a, b, 0, j, l, m)=M+\left(M^{2}-2 a b+\left(2 v_{ \pm}+1\right) /(a+1)\right)^{1 / 2}-\left(\Delta E_{c c}(0, j, l, s, m)\right)^{1 / 2} \\
& E_{n c}^{(1)}(a, b, 1, j, l, m)=M+\left(M^{2}-2 a b+\left(2 v_{ \pm}+3\right) /(a+1)\right)^{1 / 2}-\left(\Delta E_{c c}(1, j, l, s, m)\right)^{1 / 2} \\
& E_{n c}^{(n)}(a, b, p, j, l, m)=M \pm\left(M^{2}-2 a b+\left(2 p+2 v_{ \pm}+1\right) /(a+1)\right)^{1 / 2}-\left(\Delta E_{c c}(p, j, l, s, m)\right)^{1 / 2}
\end{aligned}
$$

\section{NEW MASSES OF QUARKONIUM SYSTEM IN RELATIVISTIC NC QUANTUM MECHANICS SYMMETRIES}

Now, we want to apply Eq. (28) on the bosonic particles like heavy-light $Q \bar{q}$, $(Q=c, q=u / d, s)$ mesons, and the quarkonium system $q \bar{q},(q=c, b, s)$ with a non-null spin that have the quark and antiquark flavor, it is well known that the spin of charmonium and bottomonium equal two values ( 0 or 1$)$ because it is a consisted of quark and anti-quark. For spin-one, we have $|l-1| \leq j \leq|l+1|$, thus we have three values of $\left(j_{1}=l+1, j_{2}=l, j_{3}=l-1\right)$, allows us the corresponding three values $\left(k_{1}(l), k_{2}(l), k_{3}(l)\right) \equiv \frac{1}{2}(l,-2,-2 l-2)$ and thus, we three values of energy:

$$
\begin{gathered}
E_{n c}^{c c}\left(k_{1}(l), a, b, n, j=l+1, l, m\right)=M \pm\left(M^{2}-2 a b+\left(2 p+2 v_{ \pm}+1\right) /(a+1)\right)^{1 / 2}+\left[\Xi\left(\frac{l}{2} \Theta+\aleph \sigma m\right)\right]^{1 / 2} \\
E_{n c}^{c c}\left(k_{2}(l), a, b, n, j=l, l, m\right)=M \pm\left(M^{2}-2 a b+\left(2 p+2 v_{ \pm}+1\right) /(a+1)\right)^{1 / 2}+[\Xi(-\Theta+\aleph \sigma m)]^{1 / 2}(30 . \\
E_{n c}^{c c}\left(k_{3}(l), a, b, n, j=l-1, l, m\right)=M \pm\left(M^{2}-2 a b+\left(2 p+2 v_{ \pm}+1\right) /(a+1)\right)^{1 / 2} \\
+\left[\Xi\left(-\frac{l+1}{2} \Theta+\aleph \sigma m\right)\right]^{1 / 2}
\end{gathered}
$$

with $\Xi(p, j, l, s, m)=l(l+1)\left\langle r^{-4}\right\rangle_{(p, l, m)}-\left(E_{p}+M\right)\left(i a\left\langle r^{-1}\right\rangle_{(p, l, m)}-b\left\langle r^{-3}\right\rangle_{(p, l, m)}\right)$

The mass of the heavy-light $Q \bar{q},(Q=c, q=u / d, s)$ mesons, and the quarkonium system $q \bar{q}, \quad(q=c, b, s)$ can be obtained in the symmetries of ordinary quantum 
mechanics by applying the following formula ${ }^{5-6,54-55}$ :

$$
M=\left\{\begin{array}{cc}
2 m_{q}+E_{p l} & \text { for }: \bar{c}, b \bar{b} \\
m_{q}+m_{q}+E_{p l} & \text { for }: \bar{s}, c \bar{u} \text { and } \bar{s} \bar{c}
\end{array}\right.
$$

Here $m_{q}$ are bare quark masses for heavy-light $Q \bar{q},(Q=c, q=u / d, s)$ mesons and the quarkonium system $q \bar{q},(q=c, b, s)$ and $M$ denote to the mass the charmonium $c \bar{c}$, bottomonium $b \bar{b}$, charmonium $c \bar{c}, \quad s \bar{s}$ and mesons $c \bar{s}$ in the relativistic quantum mechanics under ordinary complex potential. Thus, the modified mass of heavylight $Q \bar{q}, \quad(Q=c, q=u / d, s)$ mesons and the quarkonium system $q \bar{q}$, $(q=c, b, s)$ become as follows:

$$
M_{n c}^{c c}(\mathrm{~S}=1)=\left\{\begin{array}{l}
2 m_{q}+\frac{1}{3} \sum_{i=1}^{3} E_{n c}^{c c}\left(k_{i}(l), a, b, n, j_{i}, l, m\right) \text { for }: c \bar{c}, b \bar{b} \text { and } \bar{s} \\
m_{q}+m_{q}+\frac{1}{3} \sum_{i=1}^{3} E_{n c}^{c c}\left(k_{i}(l), a, b, n, j_{i}, l, m\right) \text { for }: c \bar{s}, c \bar{u} \text { and } c \bar{d}
\end{array}\right.
$$

Thus, the modified masse of the heavy quarkonium system the heavy-light $Q \bar{q}$, $(Q=c, q=u / d, s)$ mesons and the quarkonium system $q \bar{q}, \quad(q=c, b, s)$ become as follows:

$$
M_{n c}^{c c}(\mathrm{~S}=1)= \begin{cases}2 m_{q}+E_{p l}+\delta M_{n c}^{c c} & \text { for }: c \bar{c}, b \bar{b} \text { and } s \bar{s} \\ m_{q}+m_{q}+\delta M_{n c}^{c c} & \text { for }: c \bar{s}, c \bar{u} \text { and } c \bar{d}\end{cases}
$$

While the modified masses $\delta M_{n c}^{c c}$ is given by:

$$
\delta M_{n c}^{c c} \equiv \frac{1}{3}\left[\Xi\left\{\frac{l}{2} \Theta+\aleph \sigma m\right\}\right]^{1 / 2}+[\Xi\{-\Theta+\aleph \sigma m\}]^{1 / 2}+\left[\Xi\left\{-\frac{l+1}{2} \Theta+\aleph \sigma m\right\}\right]^{1 / 2}
$$

For the spin-zero case, $j=l$ equal only one value, which allows us to obtain a null value for the parameter $k(j, l, s)$, thus the modified mass of the quarkonium system $M_{n c}^{c c}$ can be determined according to the following new generalized formula:

$M_{n c}^{c c}(\mathrm{~S}=0)=\left\{\begin{array}{l}2 m_{q}+E_{r-n c}^{c c}(a, b, n, j=l, l, S=0, m) \text { for }: c \bar{c}, b \bar{b} \text { and } \bar{s} \\ m_{q}+m_{q}++E_{r-n c}^{c c}(a, b, n, j=l, l, S=0, m) \text { for }: c \bar{s}, c \bar{u} \text { and } c \bar{d}\end{array}\right.$

which gives

$M_{n c}^{c c}(\mathrm{~S}=0)= \begin{cases}2 m_{q}+E_{p l}+\left[\Delta E_{c c}(n, j, l, s, m) \aleph \sigma m\right]^{1 / 2} & \text { for }: c \bar{c}, b \bar{b} \text { and } s \bar{s} \\ m_{q}+m_{q}+E_{p l}+\left[\Delta E_{c c}(n, j, l, s, m) \aleph \sigma m\right]^{1 / 2} & \text { for }: c \bar{s}, c \bar{u} \text { and } c \bar{d}\end{cases}$

On the other hand, it is evident to consider the quantum number $m$ takes $(2 l+1)$ values and we have also two values for $j=l \pm 1, l$, thus any state in ordinary 3 -dimensional space of the energy for heavy-light $Q \bar{q},(Q=c, q=u / d, s)$ mesons, and the quarkonium 
system $q \bar{q},(q=c, b, s)$ with spin-1 under the modified complex central potential will become triplet $3(2 l+1)$ sub-states. To obtain the total complete degeneracy of energy level of the modified complex potential in the symmetries of the relativistic threedimensional noncommutative quantum mechanics, we will have to sum for all allowed values of angular momentum quantum number $l=0, \overline{p-1}$. Total degeneracy is thus,

$$
\underbrace{2 \sum_{l=0}^{p-1}(2 l+1) \equiv 2 n^{2}}_{\mathrm{RQM}} \rightarrow \underbrace{3 \sum_{l=0}^{p-1} 2(2 l+1) \equiv 6 n^{2}}_{\mathrm{RNCQM}}
$$

The degeneracy of the initial spectral is broken and replaced by a new more precise and clear one. The doubled the total complete degeneracy of energy level for the heavylight $Q \bar{q},(Q=c, q=u / d, s)$ mesons and the quarkonium system $q \bar{q},(q=c, b, s)$ with spin-1, inrelativistic noncommutative quantum mechanics symmetries under the modified complex potential, gives very clear physical indicator shows that physical treatments with relativistic noncommutative quantum mechanics appear more detailed and clarity if it compared with similar energy levels obtained in ordinary relativistic quantum mechanics.

It should be noted that the appearance of the spin-orbit interaction with the expression $V_{\text {pert }}^{c c}(r)=f(r) \overrightarrow{\mathbf{L}} \vec{S}$ (here $f(r)=\frac{l(l+1)}{r^{4}}-(E+M)\left(\frac{i a}{r}+\frac{b}{r^{3}}\right)$ ) gives a physical indicator to extend the Klein-Gordon equation under central complex potential to the modified Klein-Gordonrelativistic three-dimensional noncommutative quantum mechanics equation under modified central complex potential to include bosonic particles with

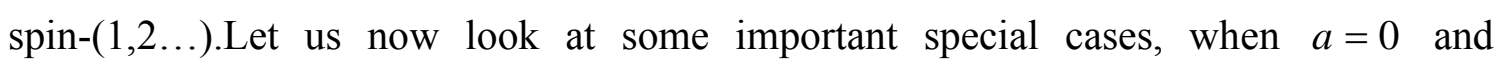
$b=-Z e^{2}$, where we conclude the effective Colombian potential in the symmetries of relativistic noncommutative three-dimensional real space $V_{\text {pert }}^{\text {col }}\left(r, a=0, b=-Z e^{2}\right)$ and the corresponding like radial Schrödinger equation which exactly compatible with the results obtained in Ref. [25]:

$$
V_{\text {pert }}^{c o l}\left(r, a=0, b=-Z e^{2}\right)=\left\lfloor\frac{l(l+1)}{r^{4}}+(E+M) \frac{Z e^{2}}{r^{3}}\right\rfloor \overrightarrow{\mathbf{L}} \vec{\Theta}
$$

and

$$
\left(\begin{array}{l}
\frac{d^{2}}{d r^{2}}+\left(E_{n l}^{2}-M^{2}\right)-2\left(E_{n l}+M\right)\left(-\frac{Z e^{2}}{r}\right)-\frac{l(l+1)}{r^{2}} \\
-\left[\frac{l(l+1)}{r^{4}}+\left(E_{n l}+M\right) \frac{Z e^{2}}{r^{3}}\right] \overrightarrow{\mathbf{L}} \vec{\Theta}
\end{array}\right) U_{n l}(r)=0
$$


Regarding obtained results in Equations. (38) and (39), the energy shift is depended on the spin non zero (spin-1) can conclude that the modified Klein-Gordon equation which treated in our paper under the modified complex potential can be prolonged to describe not only spin-zero particles, but particles with spin-1, for example, the heavy-light $Q \bar{q}$, $(Q=c, q=u / d, s)$ mesons, and the quarkonium system $q \bar{q},(q=c, b, s)$. Thus, one can conclude that the modified Klein-Gordon equation becomes similar to the DuffinKemmer equation, which describes bosonic particles with spin non-null. It should be noted that our current results are an excellent agreement with our previously published work, particularly for example the new modified potential containing Cornell, Gaussian, and inverse square terms ${ }^{56}$, modified quark-antiquark interaction potential ${ }^{57}$ and modified Cornell plus inverse quadratic potential ${ }^{58}$. Furthermore, and in a general way, the comparisons show that our results are in very good agreement with reported works ${ }^{25-29}$. Worthwhile it is better to mention that for the two simultaneously limits $(\Theta, \sigma) \rightarrow(0,0)$, we recover the results of the commutative space obtained in Ref.[13] For the modified central complex potential, this means that our present calculations are correct.

\section{CONCLUSION}

This section of our paper gives a summary of the basic points in our work; we have investigated the modified Klein-Gordon equation for modified central complex potential in the relativistic noncommutative three-dimensional spaces. The energy levels of the ground state, the first excited state and $p^{\text {th }}$ excited state $\left(E_{n c}^{(0)}(a, b, 0, j, l, m)\right.$, $\left.E_{n c}^{(1)}(a, b, 1, j, l, m), E_{n c}^{(n)}(a, b, n, j, l, m)\right)$ as functions of the shift energy $\left(\Delta E_{c c}(0, j, l, s, m)\right.$, $\left.\Delta E_{c c}(1, j, l, s, m), \Delta E_{c c}(p, j, l, s, m)\right)$ and $\left(E_{0 l}, E_{1 l}, E_{p l}\right)$, is obtained via first-order perturbation theory and expressed bythe parabolic cylinder functions, Gamma function, the discreet atomic quantum numbers $(j, l, s, m)$ and the potential parameters ( $a$ and $b)$, in addition to the noncommutativity parameters $(\Theta$ and $\sigma)$. This behavior is similar to the perturbed both perturbed new modified Zeeman effect and perturbed spin-orbit coupling in which an external magnetic field is applied to the system and the spin-orbit couplings which are generated with the effect of the perturbed effective potential $V_{p e r t}^{c c}(r)$ in the symmetries of relativistic three-dimensional noncommutative quantum mechanics. We have seen that the physical treatment of modified Klein-Gordon equation under the modified central complex potential for bosonic particles like heavy-light $Q \bar{q}$, $(Q=c, q=u / d, s)$ mesons and the quarkonium system $q \bar{q},(q=c, b, s)$ with spin- $(0,1)$ gives a very clear physical indicator show that physical treatments with relativistic noncommutative quantum mechanics appear more detailed and clarity if it compared with similar energy levels obtained in ordinary relativistic quantum mechanics. Thus, we can conclude that the modified Klein-Gordon equation becomes similar to the DuffinKemmer equation under the modified central complex potential, it can describe a dynamic state of a particle with spin one in the symmetries of relativistic noncommutative quantum mechanics. The results related to relativistic quantum mechanics under the central complex potentialbecomes a particular case when we make the two simultaneously limits $(\Theta, \sigma) \rightarrow(0,0)$. The comparisons show that our theoretical 
results are in very good agreement with reported works. Finally, we can conclude the important results from this article, are the ability of the modified Klein-Gordon equation on playing a vital role in more profound interpretations in describing elementary particles such as the heavy-light $Q \bar{q},(Q=c, q=u / d, s)$ mesons and the quarkonium system $q \bar{q}, \quad(q=c, b, s)$ at high-energy physics under the modified central complex potential.

\section{ACKNOWLEDGMENTS}

Anonymous referees are acknowledged for their useful comments and suggestions which greatly improved the paper.

\section{REFERENCES}

1. R. Kumar and F. Chand, Series solutions to the N-dimensional radial Schrödinger equation for the quark-antiquark interaction potential. Phys. Scr. 85 (2012) 055008. DOI:https://doi.org/10.1088/0031-8949/85/05/055008

2. R. Kumar and F. Chand, Asymptotic Study to the N-Dimensional Radial Schrödinger Equation for the Quark-Antiquark System.Commun. Theor. Phys. 59, (2013) 528532.DOI:http://iopscience.iop.org/0253-6102/59/5/02

3. M.Abu-Shady, Heavy Quarkonia and $\overline{b c}$ Mesons in the Cornell Potential with Harmonic Oscillator Potential in the N-dimensional Schrödinger Equation.International Journal of Applied Mathematics and Theoretical Physics.2(2), (2016) 1620.DOI:10.11648/j.ijamtp.20160202.11

4. V.Kher, N.Devlaniand R. K. Kumar,Spectroscopy, decay properties and Regge trajectories of the $B$ and Bs mesons.Chinese Physics C.41(9),(2017) 093101.DOI: 10.1088/1674$\underline{1137 / 41 / 9 / 09310}$

5. M.Abu-Shady andE. M.Khokha, Heavy-Light Mesons in the Nonrelativistic Quark Model Using Laplace Transformation Method. Advances in High Energy Physics.(2018) 112.DOI:https://doi.org/10.1155/2018/7032041

6. M. Abu-Shady and S.Y. Ezz-Alarab. Trigonometric Rosen-Morse Potential as a QuarkAntiquark Interaction Potential for Meson Properties in the Non-relativistic Quark Model Using EAIM. Few-Body Systems. 60(4), (2019). DOI: 10.1007/s00601-019-1531-y.

7. Abdelmadjid Maireche, Heavy-light mesons in the symmetries of extended nonrelativistic quark model. Yanbu Journal of Engineering and Science YJES. 17, (2019) 51-63.

8. N.V. Maksimenko and S.M.Kuchin. Electric polarizability of pions in semirelativistic quark model. Physics of Particles and Nuclei Letters. 9(2), (2012) 134-138. Doi: 10.1134/s1547477112020112.

9. Jing-Bin Liu and Mao-Zhi Yang, Heavy-light mesons in a relativistic model. Chinese Physics C. 40(7), (2016) 073101. DOI: 10.1088/1674-1137/40/7/073101

10. A. Martin, A simultaneous fit of b, c,s (bcs Pairs) and c spectra. Physics Letters B.100(6), (1981) 511-514. DOI:10.1016/0370-2693(81)90617-1

11. Ö. Yeşiltaş, M. Şimşek, R. Sever andC. Tezcan, Exponential Type Complex and NonHermitian Potentials in PT-Symmetric Quantum Mechanics. Physica Scripta.67(6), (2003) 472-475.DOI:10.1238/physica.regular.067a00472 
12. M. Simsek and H. Egrifes, The Klein-Gordon equation of generalized Hulthén potential in complex quantum mechanics. Journal of Physics A: Mathematical and General. 37(15), (2004) 4379-4393. DOI:10.1088/0305-4470/37/15/007

13. V.K. Srivastava, S.K. Bose. Exact solution of relativistic Schrodinger equation for the central complex potential V $(r)=i a r$ plus (b/r). Indian J.Pure Appl.Phys. 47, (2009) 547550.DOI:http://hdl.handle.net/123456789/5460

14. Virendrasinh Kher et al,Excited-state mass spectra, decay properties and Regge trajectories of charm and charm-strange mesons.Chinese Phys. C.41 (2017) 073101. DOI:https://doi.org/10.1088/1674-1137/41/7/073101

15. S. Capozziello, G. Lambiase and G. Scarpetta, Generalized Uncertainty Principle from QuantumGeometry. Int. J. Theor. Phys. 39 (2000) 15. DOI: https://doi.org/10.1023/A:1003634814685

16. E. Passos, L.R. Ribeiro and C. Furtado, Noncommutative Anandan quantum phase. Phys Rev A.76 (2007) 012113. DOI:https://doi.org/10.1103/PhysRevA.76.012113

17. L.R. Ribeiro, E. Passos, C. Furtado and J.R. Nascimento,Geometric phases modified by a Lorentz-symmetry violation background.International Journal of Modern Physics A, 30(14) (2015) 1550072. DOI: https://doi.org/10.1142/S0217751X15500724

18. Ö. F. Dayi, Dynamics of dipoles and quantum phases in noncommutative coordinates, EPL (Europhysics Letters).85(4) (2009) 41002.DOI: https://doi.org/10.1209/0295-5075/85/41002

19. Ö. F. Dayi and B. Yapışkan, An Alternative Formulation of Hall Effect and Quantum Phases in Noncommutative Space. Physics Letters A.374(37) (2010) 3810-3817. DOI: 10.1016/j.physleta.2010.07.043

20. A. Jamel, Heavy quarkonia with Cornell potential on noncommutative space.Journal of Theoretical and Applied Physics, 5-1(2011) 21-24.

21. N. Chair, and M.A. Dalabeeh, The noncommutative quadratic Stark effect for the H-atom. Journal of Physics A: Mathematical and General, 38(7) (2005) 1553-1558.DOI: https://doi.org/10.1088/0305-4470/38/7/010

22. W. Heisenberg. Letter to R. Peierls (1930), in 'Wolfgang Pauli, Scientific Correspondence', Vol. III, p.15, Ed. K. von Meyenn - 1985. Springer: Verlag.

23. H. Snyder, Quantized Space-Time. Phys. Rev.71(1), (1947) 3841.DOI:10.1103/physrev.71.38

24. YUAN Yi, LI Kang, WANG Jian-Hua and CHEN Chi-Yi. Spin-1/2 relativistic particle in a magnetic field in NC phase space. Chinese Physics C. 34(5), (2010) 543547. DOI:10.1088/1674-1137/34/5/005

25. H. Motavalli and A.R. Akbarieh, Klein-Gordon equation for the Coulomb potential in noncommutative space. Modern Physics Letters A. 25(29), (2010) 2523-2528. DOI:10.1142/s0217732310033529 
26. M. Darroodi, H. Mehraban and S. Hassanabadi, The Klein-Gordon equation with the Kratzer potential in the noncommutative space. Modern Physics Letters A. (2018) 1850203. DOI:10.1142/s0217732318502036

27. H. Hassanabadi, S.S. Hosseini and Z. Molaee, Klein Gordon Oscillators in Commutative and Noncommutative Phase Space with Psudoharmonic Potential in the Presence and Absence Magnetic Field. Communications in Theoretical Physics. 60(1), (2013) 9-18. DOI:10.1088/0253-6102/60/1/02

28. Abdelmadjid Maireche, The Klein-Gordon Equation with Modified Coulomb Potential Plus Inverse-Square-Root Potential in Three-Dimensional Noncommutative Space. To Physics Journal. 3, (2019) 186-196. https://purkh.com/index.php/tophy/article/view/489

29. Abdelmadjid Maireche, The Klein-Gordon equation with modified Coulomb plus inversesquare potential in the noncommutative three-dimensional space. Modern Physics Letters A. 35(5), (2020) 2050015. DOI:10.1142/s0217732320500157

30.P.M. Ho and H.C. Kao, NoncommutativeQuantum Mechanics from Noncommutative Quantum Field Theory. Physical Review Letters 88(15), (2002). DOI:10.1103/physrevlett.88.151602

31. B. Mirza and M. Zarei, Non-commutative quantum mechanics and the Aharonov-Casher effect. The European Physical Journal C-Particles and Fields. 32(4), (2004) 583586. DOI:10.1140/epjc/s2003-01522-8

32. K.P. Gnatenko, Parameters of noncommutativity in Lie-algebraic noncommutative space. Physical Review D. 99(2), 026009-1(2019). DOI:10.1103/physrevd.99.026009

33. K.P. Gnatenko and V.M. Tkachuk, Weak equivalence principle in noncommutative-2469 (2017) phase space and the parameters of noncommutativity. Physics Letters A. 381(31), 2463. DOI:10.1016/j.physleta.2017.05.056

34. O. Bertolami, J. G. Rosa, C.M.L. De aragao, P. Castorina andD. Zappala, Scaling ofvariables and the relation between noncommutativeparameters in noncommutative quantum mechanics. Modern Physics Letters A 21(10)(2006) 795-802. DOI:10.1142/s0217732306019840

35. S.A. Alavi, Hyperfine splitting in noncommutative spaces. Physica Scripta78(1), (2008) 015005. DOI:10.1088/0031-8949/78/01/015005

36. Abdelmadjid Maireche, A Recent Study of Excited Energy Levels of Diatomics for Modified more General Exponential Screened Coulomb Potential: Extended Quantum Mechanics. J. Nano- Electron. Phys. 9(3), (2017) 03021.DOI 10.21272/jnep.9(3).03021

37. A.E.F. Djemaïand H. Smail, On Quantum Mechanics on Noncommutative Quantum Phase Space. Commun. Theor. Phys. (Beijing, China) 41(6), (2004) 837-844. DOI:10.1088/0253$\underline{6102 / 41 / 6 / 837}$

38. O. Bertolami and P. Leal, Aspects of phase-space noncommutative quantum mechanics. Physics Letters B 750, (2015) 6-11. DOI:10.1016/j.physletb.2015.08.024

39. Abdelmadjid Maireche. A. New Relativistic Atomic Mass Spectra of Quark ( $u, d$ and s) for Extended Modified Cornell Potential in Nano and Plank's Scales. J. Nano- Electron. Phys. 8(1), 01020-1 - 01020-7 (2016). DOI: 10.21272/jnep.8(1).01020

40. Abdelmadjid Maireche, New Bound State Energies for Spherical Quantum Dots in Presence of a Confining Potential Model at Nano and Plank's Scales. NanoWorld J. 1(4), (2016) 122129. DOI: $10.17756 /$ nwj.2016-016 
41. D. Jido and M.Sakashita. Quark confinement potential examined by excitation energy of the $c$ and $b$ baryons in a quark-diquark model.Prog. Theor. Exp. Phys. (2016) 083D02 (15 pages). DOI: $10.1093 / \mathrm{ptep} / \mathrm{ptw} 113$

42. S. M. Kuchin and N. V. Maksimenko. Theoretical Estimations of the Spin Averaged Mass Spectra of Heavy Quarkonia and Bc Mesons. Univ. J. Phys. Appl. 1 (2013) 295.DOI:10.13189/ujpa.2013.010310

43. Abdelmadjid Maireche,Nonrelativistic treatment of Hydrogen-like and neutral atoms subjected to the generalized perturbed Yukawa potential with centrifugal barrier in the symmetries of noncommutative Quantum mechanics. International Journal of Geometric Methods in Modern Physics. 17(05), (2020) 2050067. DOI:10.1142/S021988782050067X

44. Abdelmadjid Maireche, New Bound States for Modified Vibrational-Rotational Structure of Supersingular Plus Coulomb Potential of the Schrödinger Equation in One-Electron Atoms. International Letters of Chemistry, Physics and Astronomy 73, (2017) 31-45. DOI:https://doi.org/10.18052/www.scipress.com/ILCPA.73.31

45. Abdelmadjid Maireche, Extended of the Schrödinger Equation with New Coulomb Potentials plus Linear and Harmonic Radial Terms in the Symmetries of Noncommutative Quantum Mechanics. J. Nano- Electron. Phys. 10(6), (2018) 06015-1 - 06015-7. DOI: https://doi.org/10.21272/jnep.10(6).06015

46. Abdelmadjid Maireche, Investigations on the Relativistic Interactions in One-Electron Atoms with Modified Yukawa Potential for Spin 1/2 Particles. International Frontier Science Letters 11,(2017) 29-44. DOI: https://doi.org/10.18052/www.scipress.com/IFSL.11.29

47. C. Bastos, O. Bertolami, N.C. Dia and J.N. Prata, Weyl-Wigner formulation of noncommutative quantum mechanics. Journal of Mathematical Physics. 49(7), (2008) 072101. DOI:10.1063/1.2944996

48. Zhang, J. Fractional angular momentum in non-commutative spaces, Physics Letters B 584(1-2), 204-209 (2004). DOI:10.1016/j.physletb.2004.01.049

49. J. Gamboa, M. Loewe, and J.C. Rojas, Noncommutative quantum mechanics. Phys. Rev. D. (2001) 64 067901. DOI: https://doi.org/10.1103/PhysRevD.64.067901.

50. M. Chaichian, Sheikh-Jabbari and A. Tureanu, Hydrogen Atom Spectrum and the Lamb Shift in Noncommutative QED. Physical Review Letters. 86(13), (2001) 2716-2719. DOI:10.1103/physrevlett.86.2716.

51. J. Wang and $\mathrm{K}$. Li, The HMW effect in noncommutative quantum mechanics. Journal of Physics A: Mathematical and Theoretical 40(9), (2007) 2197-2202.doi:10.1088/1751$\underline{8113 / 40 / 9 / 021}$

52. K. Li, and J. Wang, The topological AC effect on non-commutative phase space. The European Physical Journal C. 50(4), (2007) 1007-1011. DOI:10.1140/epjc/s10052-007$\underline{0256-0}$

53. I.S. Gradshteyn and I.M.Ryzhik, Table of Integrals, Series and Products, edited by Alan Jeffrey (the University of Newcastle upon Tyne, England and Daniel Zwillinger, Rensselaer Polytechnic Institute USA 2007), 7th. Ed p. 365.

54. S.M. Kuchin and N.V.Maksimenko, Theoretical Estimations of the Spin - Averaged Mass Spectra of Heavy Quarkonia and Bc Mesons. Universal Journal of Physics and Application 1(3), (2013) 295-298. DOI: 10.13189/ujpa.2013.010310

55. M. Abu-Shady, T.A. Abdel-Karim and E.M. Khokha, Exact Solution of the N-dimensional radial Schrödinger equation via Laplace transformation method with the generalized Cornell potential. SF J. Quantum Phys. 2, (2018) 1000017. 
56. Abdelmadjid Maireche, A New Model for Describing Heavy-Light Mesons in The Extended Nonrelativistic Quark Model Under a New Modified Potential Containing Cornell, Gaussian And Inverse Square Terms in The Symmetries Of NCQM. To Physics Journal. 3, (2019) 197-215.https://purkh.com/index.php/tophy/article/view/500

57. Abdelmadjid Maireche,A New Asymptotic Study to the 3-Dimensional Radial Schrödinger Equation under Modified Quark-antiquark Interaction Potential. J Nanosci Curr Res 4:131(2019).

58. Abdelmadjid Maireche,A theoretical investigation of nonrelativistic bound state solution at finite temperature using the sum of modified Cornell plus inverse quadratic potential. Sri Lankan Journal of Physics, Vol. 21 (2020) 11-35K. DOI: http://DOI.org/ 10.4038/sljp.v20i0. 